\title{
Violência cometida por pessoa conhecida - Brasil, 2013
}

\author{
Violence committed by acquaintances - Brazil, 2013
}

\author{
Márcio Dênis Medeiros Mascarenhas ${ }^{1}$ \\ Raniela Borges Sinimbu ${ }^{1}$ \\ Deborah Carvalho Malta ${ }^{2}$ \\ Marta Maria Alves da Silva ${ }^{3}$ \\ Alexandre Fonseca Santos ${ }^{4}$ \\ Maria Lucia França Pontes Vieira ${ }^{5}$ \\ Célia Landmann Szwarcwald ${ }^{6}$
}

${ }^{1}$ Programa de PósGraduação em Saúde e Comunidade, Universidade Federal do Piauí. Av. Frei Serafim, 2280. 64000020 Teresina PI Brasil. mdm.mascarenhas@ gmail.com

${ }^{2}$ Departamento de Enfermagem MaternoInfantil e Saúde Pública, Universidade Federal de Minas Gerais. Belo Horizonte MG Brasil. ${ }^{3}$ Universidade Federal de Goiás. Goiânia GO Brasil. ${ }^{4}$ Ministério da Saúde. Brasília DF Brasil.

${ }^{5}$ Fundação Instituto Brasileiro de Geografia e Estatística. Rio de Janeiro RJ Brasil.

${ }^{6}$ Instituto de Comunicação e Informação Científica e Tecnológica em Saúde, Fiocruz. Rio de Janeiro RJ Brasil.
Abstract The scope of this article is to describe the prevalence of violence committed by acquaintances in accordance with demographic characteristics. It is a descriptive study, based on data from a population-based National Health Research survey conducted in Brazil in 2013. Data from the adult population ( $\geq 18$ years) of 64,348 households. Prevalence rates were calculated and their respective 95\% confidence intervals (95\%CI) according to gender, age, education, race/color, area of residence and geographical region. The prevalence of violence committed by acquaintances was $2.5 \%$ (95\%CI 2.3-2.7), significantly higher in women (3.1\%; 95\%CI 2.8-3.5) compared to men (1.8\%; 95\% CI 1.6-2.1). This prevalence was higher in the population aged 18-29 years old (3.2\%; 95\%CI 2.8-3.7) compared to older individuals (1.1\%; 95\%CI 0.8-1.3), and among residents of the North (3.2\%; 95\%CI 2.5-3.8) and Northeast Regions (3.0\%; 95\%CI 2.5-3.8) when compared to residents of the Southeast Region (2.0\%; 95\%CI 1.6-2.3). Violence was present in higher prevalence among women, proving the occurrence of gender-based violence and confirming its occurrence in all geographical regions and in different population groups in Brazil in 2013. Key words Violence, Domestic violence, Prevalence, Health surveys, Descriptive epidemiology
Resumo O objetivo deste artigo é descrever a prevalência de violência cometida por pessoas conhecidas segundo características demográficas. Trata-se de estudo descritivo, a partir dos dados obtidos em inquérito de base populacional, a Pesquisa Nacional de Saúde, realizada no Brasil em 2013. Foram analisados dados da população adulta ( $\geq 18$ anos) em 64.348 domicílios. Calcularamse as prevalências e seus respectivos intervalos de confiança de 95\% (IC95\%) segundo sexo, faixa etária, escolaridade, cor/raça, zona de residência e região geográfica. A prevalência de violência cometida por pessoa conhecida foi de 2,5\% (IC95\% 2,3-2,7), significativamente maior nas mulheres (3,1\%; IC95\% 2,8-3,5) quando comparadas aos homens (1,8\%; IC95\% 1,6-2,1), na população jovem de 18 a 29 anos (3,2\%; IC95\% 2,8-3,7) em relação aos mais velhos (1,1\%; IC95\% 0,8-1,3) e nos residentes das Regiões Norte (3,2\%; IC95\% 2,5-3,8) e Nordeste (3,0\%; IC95\% 2,5-3,8) em comparação aos da Região Sudeste (2,0\%; IC95\% 1,6-2,3). A violência foi observada em maior prevalência no sexo feminino, comprovando a ocorrência da 'violência de gênero' e confirmando sua existência em todas as regiões geográficas e nos diferentes grupos populacionais do Brasil, em 2013. Palavras-chave Violência, Violência doméstica, Prevalência, Inquéritos epidemiológicos, Epidemiologia descritiva 


\section{Introdução}

A violência é um processo multicausal caracterizado pelo uso de força física ou poder contra um indivíduo, grupo ou comunidade, que resulte em sofrimento, morte, dano psicológico, prejuízo ao desenvolvimento ou privação. Pode expressar-se de variadas formas e atingir qualquer indivíduo independentemente de faixa etária, sexo, posição social ou etnia. Apresenta raízes históricas, culturais, econômicas e sociais ${ }^{1,2}$.

Ela pode ser subdividida em violência física, psicológica, verbal, sexual e negligência ou abandono $^{1,2}$. De maneira geral, crianças, mulheres e idosos são as principais vítimas por serem consideradas populações vulneráveis ${ }^{3}$. Por exemplo, com a transição demográfica e o envelhecimento populacional, a violência contra o idoso acompanhou a tendência crescente e sua incidência aumentou aproximadamente $150 \%$ em dez anos ${ }^{1}$.

Casos de violência contra a mulher, em geral, são banalizados, em função de aspectos culturais, devido à herança de uma sociedade machista e patriarcal, situação representada pelos atos praticados por agressores conhecidos das vítimas ou mesmo membros do ciclo familiar ${ }^{4}$. Outros segmentos populacionais, como crianças e idosos, também são vítimas de abusos e omissões que resultam em sofrimento desnecessário, violação dos direitos humanos e redução na qualidade de vida ${ }^{5}$.

A violência, por ser um fenômeno sócio-histórico, acompanhada de experiência da humanidade, torna-se problema de saúde pública porque afeta a saúde individual e coletiva, e para sua prevenção e tratamento, exige formulação de políticas específicas e organização de práticas e de serviços peculiares ${ }^{4}$.

Além disso, a violência onera o sistema de saúde devido às despesas com cuidados hospitalares, a redução dos anos de vida produtiva, a possibilidade de deixar sequelas às vítimas e o aumento da mortalidade. Percebe-se ainda que não só a vítima sofre com a agressão; a família, os cuidadores e os profissionais de saúde também são envolvidos ${ }^{4}$.

Apesar de o tema violência figurar no cenário de políticas sociais como um importante problema de saúde pública no Brasil, ainda há escassez de estudos que permitam a identificação de sua magnitude na população em geral. Alguns estudos existentes dimensionam o problema em segmentos populacionais específicos ${ }^{6,7}$, em áreas geográficas de abrangência limitada ${ }^{8}$ ou apenas em serviços selecionados ${ }^{9}$, mas não exploram a distribuição do evento em dimensão nacional.
Há limitações que dificultam conhecer a magnitude do problema, tais como: ausência de pesquisas em nível nacional que informem sobre os tipos específicos de violência e as dificuldades de estimar as incidências destes agravos por meio dos sistemas de informação e atendimentos nos serviços públicos de saúde.

Frente ao exposto, e considerando que é fundamental conhecer as características do problema para poder elaborar medidas de prevenção, o presente artigo teve como objetivo descrever os indicadores da prevalência de violência cometida por pessoas conhecidas, a partir de dados da Pesquisa Nacional de Saúde, realizada no Brasil em 2013.

\section{Métodos}

Estudo descritivo desenvolvido a partir de dados da Pesquisa Nacional de Saúde (PNS), realizada pelo Instituto Brasileiro de Geografia e Estatística (IBGE) em parceria com o Ministério da Saúde (MS). A PNS foi representativa para a população adulta ( $\geq 18$ anos) residente em domicílios permanentes, localizados em área urbana ou rural, nas cinco grandes regiões geográficas, 27 unidades da Federação (UF), capitais e municípios de cada $\mathrm{UF}^{10}$.

Adotou-se a amostragem aleatória por conglomerados em três estágios, com estratificação das unidades primárias, constituídas por setores censitários ou conjunto de setores. No segundo estágio foram selecionados os domicílios e no terceiro, sorteado um morador com idade igual ou superior a 18 anos. Houve coleta de dados em 64.348 domicílios, dos quais foram selecionados 60.202 adultos para a entrevista individual.

A coleta de dados ocorreu no período de agosto de 2013 a fevereiro de 2014, por meio do uso de computadores de mão (Personal Digital Assitance-PDA), programados para processos de crítica no preenchimento das variáveis e operados por entrevistadores treinados. Informações detalhadas sobre a pesquisa podem ser obtidas em publicações específicas ${ }^{10,11}$.

O presente estudo incluiu os registros referentes aos indivíduos que responderam positivamente à pergunta: "Nos últimos 12 meses, o(a) senhor(a) sofreu alguma violência ou agressão de pessoa conhecida [como pai, mãe, filho(a), cônjuge, parceiro(a), namorado(a), amigo(a), vizinho(a)]?", o que permitiu calcular os seguintes indicadores:

a) Proporção (\%) de adultos que referiram ter sofrido alguma violência ou agressão de pes- 
soa conhecida nos últimos 12 meses: número de indivíduos que referiram ter sofrido alguma violência ou agressão de pessoa conhecida nos últimos 12 meses dividido pelo total de entrevistados;

b) Proporção (\%) de adultos que sofreram violência ou agressão de pessoa conhecida nos últimos 12 meses e que deixaram de realizar quaisquer de suas atividades habituais devido a estas: número de indivíduos que deixaram de realizar quaisquer de suas atividades habituais devido à violência ou agressão cometida por pessoa conhecida dividido pelo número de indivíduos que referiram ter sofrido alguma violência ou agressão de pessoa conhecida nos últimos 12 meses;

c) Proporção (\%) de adultos que sofreram violência ou agressão de pessoa conhecida nos últimos 12 meses que resultou em alguma lesão corporal: número de indivíduos que tiveram alguma lesão corporal devido à violência ou agressão cometida por pessoa conhecida dividido pelo número de indivíduos que referiram ter sofrido alguma violência ou agressão de pessoa conhecida nos últimos 12 meses;

d) Proporção (\%) de adultos que sofreram violência ou agressão de pessoa conhecida nos últimos 12 meses e que por esse motivo buscaram algum tipo de assistência de saúde: número de indivíduos que buscaram algum tipo de assistência de saúde devido à violência ou agressão cometida por pessoa conhecida dividido pelo número de indivíduos que referiram ter sofrido alguma violência ou agressão de pessoa conhecida nos últimos 12 meses;

e) Proporção (\%) de adultos que sofreram violência ou agressão de pessoa conhecida nos últimos 12 meses e que precisaram ser internados por esse motivo: número de indivíduos que precisaram ser internados devido à violência ou agressão cometida por pessoa conhecida dividido pelo número de indivíduos que referiram ter sofrido alguma violência ou agressão de pessoa conhecida nos últimos 12 meses;

f) Proporção (\%) de adultos que sofreram violência ou agressão de pessoa conhecida nos últimos 12 meses que tenha provocado alguma sequela ou incapacidade: número de indivíduos que tiveram alguma sequela ou incapacidade devido à violência ou agressão cometida por pessoa conhecida dividido pelo número de indivíduos que referiram ter sofrido alguma violência ou agressão de pessoa conhecida nos últimos 12 meses.

As prevalências e seus respectivos intervalos de confiança de 95\% (IC95\%) foram calculados para o total da população adulta do Brasil e de- sagregados segundo sexo (masculino; feminino), faixa etária (18 a 29; 30 a 39; 40 a 59; 60 e mais anos), nível de instrução (ensino fundamental incompleto; fundamental completo; médio completo e superior completo), cor ou raça (branca; preta; parda), zona de residência (urbana; rural), região geográfica, UFs e respectivas capitais. Não foram apresentadas as prevalências para pessoas de raça amarela e indígena, embora incluídos no total da amostra, devido ao menor número de respondentes e baixa precisão das estimativas.

As análises foram realizadas com o auxílio do programa Stata, versão 11, utilizando-se o módulo survey, adequando para amostragens complexas. Diferenças estatisticamente significativas entre as categorias foram avaliadas pela sobreposição dos intervalos de confiança.

A PNS foi aprovada pela Comissão Nacional de Ética em Pesquisa (CONEP) do Conselho Nacional de Saúde (CNS), Ministério da Saúde. Antes das entrevistas, os participantes concordaram em participar da pesquisa por meio do Termo de Consentimento Livre e Esclarecido (TCLE).

\section{Resultados}

A prevalência de violência cometida por pessoa conhecida na população adulta no Brasil em 2013 foi de 2,5\% (IC95\% 2,3-2,7), significativamente maior nas mulheres (3,1\%; IC95\% 2,8-3,5) quando comparadas aos homens (1,8\%; IC95\% 1,6-2,1). A prevalência de violência atingiu os maiores valores na população adulta jovem de 18 a 29 anos (3,2\%; IC95\% 2,8-3,7) em relação à população de idosos (1,1\%; IC95\% 0,8-1,3) e nos autodeclarados pardos (2,9\%; IC95\% 2,6-3,3) em relação aos brancos (2,1\%; IC95\% 1,8-2,4). A prevalência de violência praticada por pessoa conhecida foi relatada em maior proporção entre habitantes das regiões Norte (3,2\%; IC95\% 2,5$3,8)$ e Nordeste (3,0\%; IC95\% 2,5-3,8) quando comparados aos residentes da região Sudeste (2,0\%; IC95\% 1,6-2,3). Não foi observada diferença significativa na distribuição da prevalência de violência segundo nível de instrução e zona de residência dos entrevistados (Tabela 1).

As maiores prevalências de violência praticada por pessoa conhecida foram observadas em Sergipe, Amapá e Rio Grande do Norte, enquanto as menores foram encontradas em Rondônia, Rio de Janeiro e São Paulo. A prevalência de violência entre as mulheres foi significativamente superior à observada entre os homens no Acre, Pernambuco e Paraná (Figura 1). 
Tabela 1. Proporção de pessoas que sofreram alguma violência ou agressão cometida por pessoa conhecida nos últimos 12 meses. Brasil, 2013.

\begin{tabular}{|c|c|c|}
\hline Variáveis & $\%$ & (IC95\%) \\
\hline Total & 2,5 & $(2,3-2,7)$ \\
\hline \multicolumn{3}{|l|}{ Sexo } \\
\hline Masculino & 1,8 & $(1,6-2,1)$ \\
\hline Feminino & 3,1 & $(2,8-3,5)$ \\
\hline \multicolumn{3}{|l|}{ Faixa etária (anos) } \\
\hline $18-29$ & 3,2 & $(2,8-3,7)$ \\
\hline $30-39$ & 3,0 & $(2,5-3,4)$ \\
\hline $40-59$ & 2,5 & $(2,1-2,9)$ \\
\hline 60 e mais & 1,1 & $(0,8-1,3)$ \\
\hline \multicolumn{3}{|l|}{ Nível de instrução } \\
\hline Fundamental incompleto ${ }^{a}$ & 2,6 & $(2,3-3,0)$ \\
\hline Fundamental completo & 2,8 & $(2,3-3,3)$ \\
\hline Médio completo & 2,3 & $(1,9-2,6)$ \\
\hline Superior completo & 2,5 & $(1,9-3,2)$ \\
\hline \multicolumn{3}{|l|}{ Cor ou raça $\mathrm{a}^{\mathrm{b}}$} \\
\hline Branca & 2,1 & $(1,8-2,4)$ \\
\hline Preta & 3,1 & $(2,3-4,0)$ \\
\hline Parda & 2,9 & $(2,6-3,3)$ \\
\hline \multicolumn{3}{|l|}{ Zona de residência } \\
\hline Urbana & 2,6 & $(2,4-2,8)$ \\
\hline Rural & 2,2 & $(1,7-2,6)$ \\
\hline \multicolumn{3}{|l|}{ Região geográfica } \\
\hline Norte & 3,2 & $(2,5-3,8)$ \\
\hline Nordeste & 3,0 & $(2,5-3,8)$ \\
\hline Sudeste & 2,0 & $(1,6-2,3)$ \\
\hline Sul & 3,0 & $(2,3-3,6)$ \\
\hline Centro-Oeste & 2,6 & $(2,0-3,1)$ \\
\hline
\end{tabular}

a) Inclui os sem instrução. b) Não foram apresentadas as prevalências para pessoas de raça amarela e indígena, embora incluídos no total da amostra, devido ao menor número de respondentes e baixa precisão das estimativas. IC95\%: intervalo de confiança de $95 \%$.

Fonte: Pesquisa Nacional de Saúde, 2013.

Em Campo Grande, Curitiba, Aracaju e Macapá, a prevalência de violência foi maior entre os homens do que entre as mulheres. Por outro lado, a prevalência foi significativamente maior entre as mulheres nas capitais Porto Velho e Palmas. Dentre as capitais, Natal, Macapá, Belém e Maceió destacaram-se, apresentando prevalência superior a $4 \%$ para o total de residentes entrevistados (Figura 2).

A Tabela 2 sumariza as consequências advindas dos episódios de violência cometida por pessoa conhecida. Aproximadamente duas em cada dez vítimas (20,5\%; IC95\% 16,9-24,1) deixaram de realizar suas atividades habituais devido à violência sofrida, o que foi observado em maior proporção no sexo feminino, idade de 30 a 39 anos, baixa escolaridade, cor branca e residentes da zona rural. Proporção semelhante $(20,4 \%$; IC95\% 17,3-23,6) apresentou lesão decorrente da violência, principalmente os indivíduos com 30 a 39 anos, ensino fundamental incompleto, cor preta e parda e residente da zona rural. Dentre as vítimas de violência, 3,5\% (IC95\% 1,9-5,1) referiram ter desenvolvido alguma sequela ou incapacidade, o que foi relatado em maior proporção pelos indivíduos do sexo masculino, de 30 a 39 anos de idade e com nível médio de escolaridade. Não houve diferença significativa na distribuição das categorias das variáveis descritoras.

Quanto à utilização de serviços de saúde devido à violência, 17,9\% (IC95\% 14,5-21,2) das vítimas referiram ter recebido algum tipo de atendimento, principalmente as mulheres e as pessoas de 30 a 39 anos com ensino médio completo. Menos de 1\% (IC95\% 0,2-1,5) das vítimas necessitaram ser hospitalizadas. Não houve diferença significativa na distribuição das categorias das variáveis descritoras (Tabela 3 ).

\section{Discussão}

Os dados da PNS 2013 conseguem, pela primeira vez, apresentar um panorama geral sobre a prevalência da violência cometida por pessoas conhecidas da vítima no Brasil. O relato de que 2,5\% da população brasileira já sofreram violência ou agressão por pessoa conhecida refere-se a um contingente de 3,7 milhões de pessoas, ou seja, aproximadamente 2,4 milhões de mulheres e 1,3 milhão de homens vítimas de violência por pessoas próximas ao seu convívio familiar ou social.

Até então, o levantamento mais abrangente geograficamente sobre violência por pessoa conhecida no Brasil havia sido realizado em 2003, contemplando apenas 15 capitais brasileiras e o Distrito Federal ${ }^{12}$. Neste inquérito, limitado a investigar 6.760 mulheres de 15 a 69 anos, a prevalência de violência entre parceiros íntimos variou bastante entre as cidades, sendo mais alta nas cidades do Norte/Nordeste do que nas do Sudeste/ Sul/Centro-Oeste. A violência foi mais frequente entre casais formados por mulheres jovens e com menos anos de estudo.

A violência é um problema complexo gerador de sofrimento e que acarreta prejuízo considerável ao desenvolvimento das pessoas, das famílias, das comunidades e dos países. Tais sofrimentos que se manifestam fundamentalmente por experiências de humilhação, injustiça, desamparo 


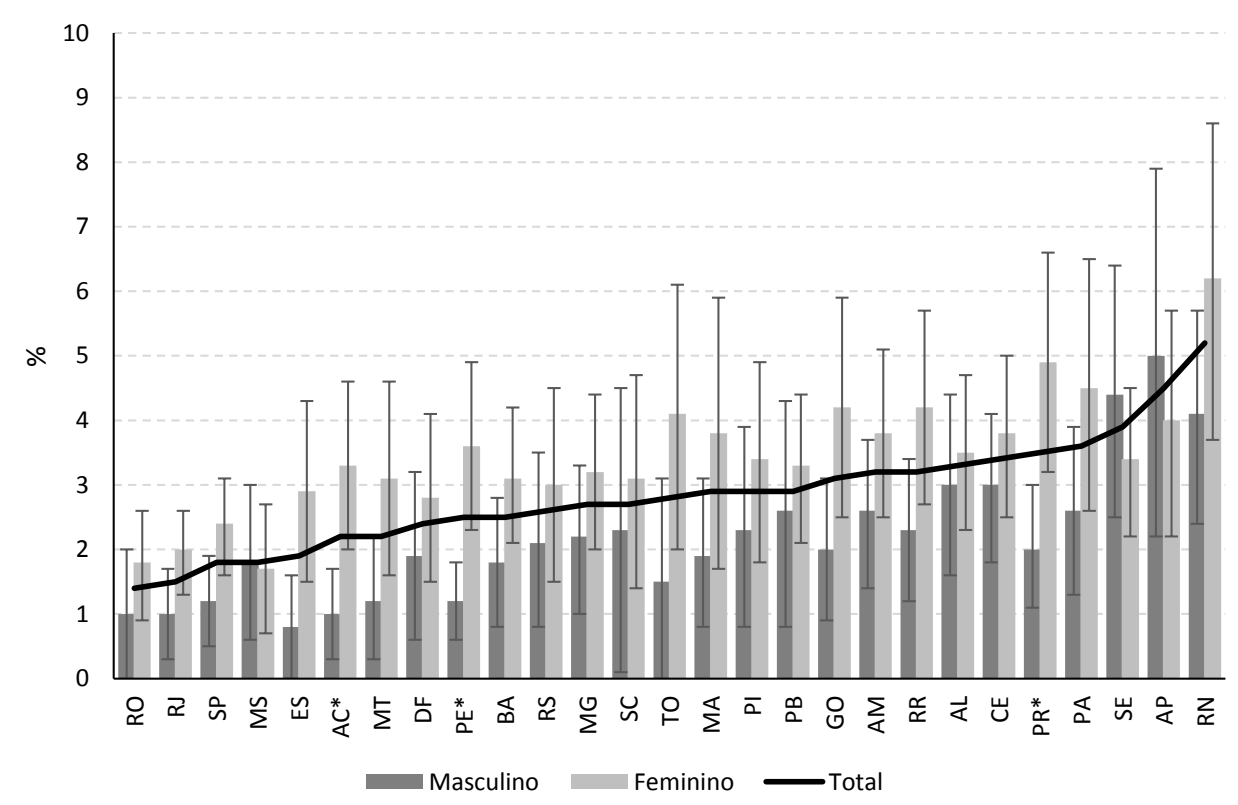

Figura 1. Proporção de pessoas que sofreram alguma violência ou agressão cometida por pessoa conhecida nos últimos 12 meses segundo Unidade Federativa e sexo. Brasil, 2013.

${ }^{*}$ Diferença estatisticamente significativa entre os sexos.

Fonte: Pesquisa Nacional de Saúde, 2013.

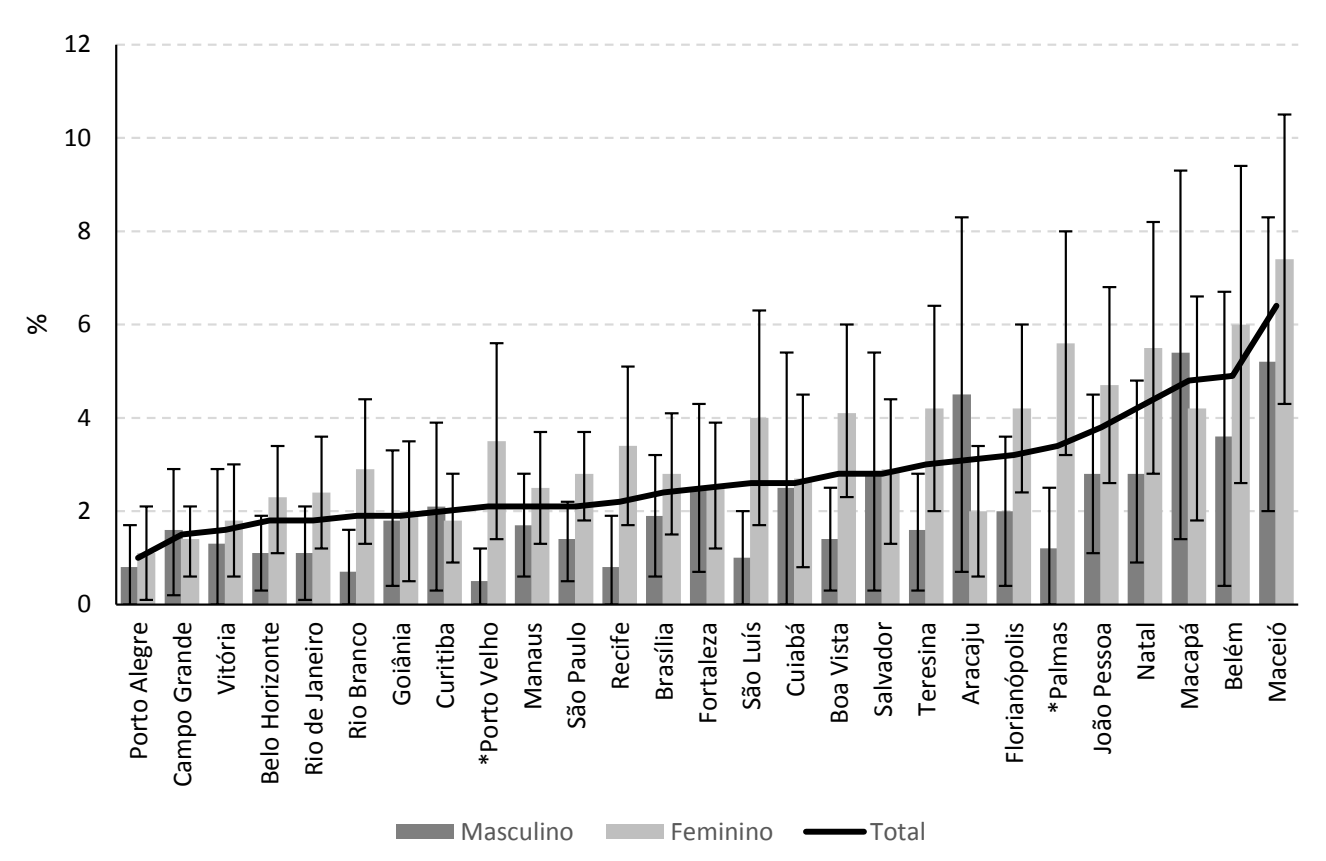

Figura 2. Proporção de pessoas que sofreram alguma violência ou agressão cometida por pessoa conhecida nos últimos 12 meses segundo capital e sexo. Brasil, 2013.

${ }^{\star}$ Diferença estatisticamente significativa entre os sexos.

Fonte: Pesquisa Nacional de Saúde, 2013. 
Tabela 2. Proporção de pessoas que relataram consequências decorrentes de violência ou agressão cometida por pessoa conhecida nos últimos 12 meses. Brasil, 2013.

\begin{tabular}{|c|c|c|c|c|c|c|}
\hline \multirow{3}{*}{ Variáveis } & \multicolumn{6}{|c|}{$\begin{array}{c}\text { Consequências decorrentes de violência ou agressão } \\
\text { por pessoa conhecida }\end{array}$} \\
\hline & \multicolumn{2}{|c|}{$\begin{array}{l}\text { Deixaram de realizar } \\
\text { atividades habituais }\end{array}$} & \multicolumn{2}{|c|}{$\begin{array}{l}\text { Tiveram lesão } \\
\text { corporal }\end{array}$} & \multicolumn{2}{|c|}{$\begin{array}{l}\text { Tiveram alguma sequel } \\
\text { ou incapacidade }\end{array}$} \\
\hline & $\%$ & (IC95\%) & $\%$ & (IC95\%) & $\%$ & (IC95\%) \\
\hline Total & 20,5 & $(16,9-24,1)$ & 20,4 & $(17,3-23,6)$ & 3,5 & $(1,9-5,1)$ \\
\hline \multicolumn{7}{|l|}{ Sexo } \\
\hline Masculino & 17,5 & $(11,4-23,5)$ & 20,0 & $(14,5-25,4)$ & 4,0 & $(0,2-7,9)$ \\
\hline Feminino & 22,1 & $(17,7-26,5)$ & 20,7 & $(16,9-24,5)$ & 3,2 & $(1,8-4,7)$ \\
\hline \multicolumn{7}{|l|}{ Faixa etária (anos) } \\
\hline $18-29$ & 19,1 & $(12,4-25,7)$ & 25,4 & $(19,6-31,2)$ & 3,4 & $(0,8-5,9)$ \\
\hline $30-39$ & 26,7 & $(18,8-34,6)$ & 26,2 & $(19,0-33,4)$ & 5,8 & $(0,7-10,9)$ \\
\hline $40-59$ & 18,4 & $(13,2-23,6)$ & 14,5 & $(10,1-18,9)$ & 2,5 & $(1,1-3,9)$ \\
\hline 60 e mais & 15,2 & $(7,6-22,8)$ & 6,1 & $(1,6-10,7)$ & 0,9 & $(0,1-1,8)$ \\
\hline \multicolumn{7}{|l|}{ Nível de instrução } \\
\hline Fundamental incompleto ${ }^{a}$ & 24,2 & $(17,8-30,6)$ & 21,9 & $(17,2-26,5)$ & 2,9 & $(1,5-4,4)$ \\
\hline Fundamental completo & 17,1 & $(10,9-23,4)$ & 20,1 & $(13,4-26,7)$ & 2,3 & $(0,8-3,9)$ \\
\hline Médio completo & 17,1 & $(11,9-22,2)$ & 21,9 & $(15,8-28,0)$ & 6,4 & $(1,4-11,3)$ \\
\hline Superior completo & 21,4 & $(9,1-33,8)$ & 13,1 & $(4,8-21,4)$ & 0,1 & $(0,0-0,3)$ \\
\hline \multicolumn{7}{|l|}{ Cor ou raça ${ }^{\mathrm{b}}$} \\
\hline Branca & 22,5 & $(16,4-28,6)$ & 15,0 & $(10,5-19,5)$ & 4,7 & $(1,0-8,5)$ \\
\hline Preta & 22,2 & $(7,7-36,8)$ & 26,3 & $(15,1-37,5)$ & 2,1 & $(0,5-3,7)$ \\
\hline Parda & 18,3 & $(14,2-22,3)$ & 23,0 & $(18,5-27,6)$ & 2,8 & $(1,4-4,3)$ \\
\hline \multicolumn{7}{|l|}{ Zona de residência } \\
\hline Urbana & 20,3 & $(16,3-24,2)$ & 19,9 & $(16,5-23,3)$ & 3,4 & $(1,6-5,1)$ \\
\hline Rural & 22,3 & $(14,2-30,3)$ & 24,5 & $(16,5-32,4)$ & 4,5 & $(1,1-8,0)$ \\
\hline \multicolumn{7}{|l|}{ Região geográfica } \\
\hline Norte & 16,7 & $(9,8-23,6)$ & 23,9 & $(17,5-30,4)$ & 1,9 & $(0,4-3,4)$ \\
\hline Nordeste & 19,3 & $(13,6-25,0)$ & 20,6 & $(16,0-25,3)$ & 4,5 & $(1,9-7,2)$ \\
\hline Sudeste & 21,2 & $(13,6-28,9)$ & 20,5 & $(13,9-27,1)$ & 2,9 & $(0,0-6,6)$ \\
\hline Sul & 24,9 & $(16,7-33,2)$ & 19,5 & $(11,3-27,7)$ & 3,3 & $(0,4-6,2)$ \\
\hline Centro-Oeste & 16,6 & $(10,8-22,4)$ & 17,2 & $(10,5-24,0)$ & 4,1 & $(0,9-7,3)$ \\
\hline
\end{tabular}

e deixam marcas psíquicas, muitas vezes não são compartilhadas socialmente ${ }^{1}$. Diversas são as situações de risco de violência dentro do lar, como histórico de agressividade na família, uso de drogas ou álcool, filhos que dependem dos pais idosos ou vice-versa ${ }^{13}$.

Quanto ao perfil da vítima, a questão do gênero é um fator preponderante na produção de violência social, interpessoal e familiar. As mulheres foram vítimas majoritárias, o que pode estar historicamente ligado à reprodução das desigualdades sociais entre homens e mulheres, situação que ainda se manifesta na sociedade atu$\mathrm{al}^{14}$. Estudos ${ }^{13,14}$ mostram que a violência contra o sexo feminino é mais frequentemente observada no ambiente doméstico.

Além disso, muitas situações de violência contra a mulher ainda permanecem veladas devido ao silêncio que muitas das vítimas adotam pelo temor a represálias ou sentimentos de humilhação, vergonha, culpa e descrédito na resolução do sistema jurídico para o problema ${ }^{13,14}$.

Merece destaque a diferença na prevalência de violência segundo sexo, evidenciando a manifestação da "violência de gênero". Cabe lembrar que essa situação ocorre apesar da recém inclusão de dispositivos como a Lei No 11.340, de 7 de agosto de 2006, conhecida como Lei Maria da 
Tabela 3. Proporção de pessoas que relataram utilização de serviços de saúde devido à violência ou agressão cometida por pessoa conhecida nos últimos 12 meses. Brasil, 2013.

\begin{tabular}{|c|c|c|c|c|}
\hline \multirow{3}{*}{ Variáveis } & \multicolumn{4}{|c|}{ Utilização de serviços de saúde } \\
\hline & \multicolumn{2}{|c|}{$\begin{array}{l}\text { Receberam algum tipo de } \\
\text { assistência de saúde }\end{array}$} & \multicolumn{2}{|c|}{$\begin{array}{c}\text { Necessitaram } \\
\text { de internação hospitalar }\end{array}$} \\
\hline & $\%$ & (IC95\%) & $\%$ & (IC95\%) \\
\hline Total & 17,9 & $(14,5-21,2)$ & 0,8 & $(0,2-1,5)$ \\
\hline \multicolumn{5}{|l|}{ Sexo } \\
\hline Masculino & 12,3 & $(7,4-17,1)$ & 1,1 & $(0,0-2,2)$ \\
\hline Feminino & 20,8 & $(16,4-25,2)$ & 0,7 & $(0,0-1,5)$ \\
\hline \multicolumn{5}{|l|}{ Faixa etária (anos) } \\
\hline $18-29$ & 17,0 & $(10,2-23,7)$ & 0,2 & $(0,0-0,5)$ \\
\hline $30-39$ & 24,0 & $(16,8-31,2)$ & 0,8 & $(0,0-2,0)$ \\
\hline $40-59$ & 16,4 & $(11,4-21,4)$ & 1,6 & $(0,0-3,2)$ \\
\hline 60 e mais & 8,3 & $(3,6-13,1)$ & 0,2 & $(0,0-0,5)$ \\
\hline \multicolumn{5}{|l|}{ Nível de instrução } \\
\hline Fundamental incompleto ${ }^{a}$ & 16,6 & $(11,1-22,2)$ & 1,2 & $(0,1-2,2)$ \\
\hline Fundamental completo & 18,6 & $(11,9-25,2)$ & 0,4 & $(0,0-0,9)$ \\
\hline Médio completo & 21,2 & $(14,5-28,0)$ & 1,0 & $(0,0-2,6)$ \\
\hline Superior completo & 12,9 & $(5,4-20,5)$ & - & - \\
\hline \multicolumn{5}{|l|}{ Cor ou raça $\mathrm{a}^{\mathrm{b}}$} \\
\hline Branca & 17,8 & $(12,5-23,0)$ & 0,6 & $(0,0-1,3)$ \\
\hline Preta & 23,0 & $(8,6-37,5)$ & 0,1 & $(0,0-0,2)$ \\
\hline Parda & 16,8 & $(12,7-20,9)$ & 1,2 & $(0,0-2,4)$ \\
\hline \multicolumn{5}{|l|}{ Zona de residência } \\
\hline Urbana & 17,7 & $(14,0-21,4)$ & 0,8 & $(0,1-1,4)$ \\
\hline Rural & 19,2 & $(12,2-26,2)$ & 1,3 & $(0,0-3,9)$ \\
\hline \multicolumn{5}{|l|}{ Região geográfica } \\
\hline Norte & 9,2 & $(4,3-14,0)$ & 0,3 & $(0,0-0,6)$ \\
\hline Nordeste & 16,8 & $(12,4-21,2)$ & 2,1 & $(0,1-4,0)$ \\
\hline Sudeste & 20,1 & $(12,4-27,7)$ & - & - \\
\hline Sul & 20,8 & $(13,5-28,2)$ & 0,4 & $(0,0-1,0)$ \\
\hline Centro-Oeste & 16,2 & $(9,0-23,5)$ & 1,1 & $(0,0-2,5)$ \\
\hline
\end{tabular}

Penha ${ }^{15}$. Esse dispositivo legal trouxe a possibilidade de instaurar medidas mais rigorosas em relação aos agressores, não havendo mais a possibilidade de julgamento das violências de gênero como crimes de menor potencial ofensivo ${ }^{16}$. Esta lei enfatizou a adoção de respostas para a criminalização da violência, com destaque na intervenção do eixo segurança-justiça, e na demanda pela criação de Delegacias Especiais de Atendimento para Mulheres e Juizados de Violência Doméstica e Familiar. Porém, sabe-se que existe uma tensão nesse setor, pois as respostas tradicionalmente oferecidas à criminalidade comum (identificação dos responsáveis, sua inculpação e consequente responsabilização com a devida pena legal) não são suficientes como respostas para o problema da violência doméstica e familiar ${ }^{17}$.

Quanto à distribuição por faixa etária, a prevalência de violência foi significativamente menor entre adultos jovens quando comparados aos idosos. Esta diferença pode ter ocorrido devido à violência ser um fenômeno pouco reconhecido e denunciado em alguns segmentos populacionais, pois ainda persistem casos em que há o segredo e a negação por parte das vítimas a fim de proteger os familiares agressores, dificultando seu conhecimento pelos sistemas de informação ${ }^{18}$.

Ressalta-se ainda que há uma maior conscientização acerca da não violência contra o idoso, devido aos recursos legais que vigoram no 
Brasil para o enfrentamento dessa situação, dentre eles os da Constituição Federal, que constitui diretrizes para a elaboração da Política Nacional de Saúde do Idoso e o Estatuto do Idoso ${ }^{5,19}$.

Quanto às regiões brasileiras, o Norte e o Nordeste, em comparação ao Sudeste, apresentaram maiores valores na prevalência de violência por pessoas conhecidas. De modo geral, estas regiões se destacaram no ranking das causas externas no Brasil, onde a violência, juntamente com os homicídios, foram os maiores responsáveis pelos anos de vida perdidos em 2008, com base em estudo sobre a carga global de doenças realizado no país ${ }^{20}$. No entanto, não se pode negar que a violência é um problema que se manifesta em todas as regiões do país, o que é mais grave. Ou seja, trata-se de um fenômeno frequente em todos os segmentos populacionais do Brasil.

No que diz respeito à busca por assistência, observou-se maior procura nos serviços de saúde por mulheres, número evidenciado também por serem elas as maiores vítimas. Além disso, Facuri et al. ${ }^{21}$ atribuem a qualidade da assistência prestada a mulheres vítimas de violência sexual, por exemplo, como fator que contribui para o aumento do número das vítimas femininas que recorrem aos serviços de saúde após situação de violência.

A violência cometida por pessoas conhecidas caracteriza-se como problema de saúde pública, principalmente quando as vítimas são populações vulneráveis como mulheres ${ }^{18} \mathrm{e}$ idosos $^{22}$. Nes- se sentido, a ênfase no cumprimento das medidas legais de proteção a estas populações e a participação do setor saúde neste contexto é importante para a prevenção da violência e promoção de saúde.

O presente estudo contribui de maneira fundamental para a melhor descrição da magnitude da violência no Brasil, mas faz-se necessário aprofundar a análise a partir de novos estudos sobre os tipos específicos, com recortes naquela cometida por parceiros íntimos e nos demais tipos de violência doméstica, por exemplo. Portanto, este é o início do monitoramento e a linha de base para novas explorações acerca dos dados obtidos na PNS 2013.

A obtenção dos resultados aqui apresentados pode ter sofrido interferência da forma de coleta dos dados. Como se tratava de pesquisa em domicílio, algumas pessoas podem ter se sentido constrangidas em revelar que sofreram violência ou, quem sabe, sentiram-se inibas por se encontrarem na presença de um agressor (a). Outra limitação da presente análise refere-se à não identificação dos tipos de agressores conhecidos (cônjuges, ex-cônjuges, familiares ou amigos) e à não classificação dos tipos de violência sofrida (sexual, verbal, física ou psicológica). Tais aspectos justificam a necessidade de aprofundar as análises futuras com os dados coletados para permitir a melhor compreensão e detalhamento da problemática da violência por pessoas conhecidas obtidos na PNS 2013.

\section{Colaboradores}

MDM Mascarenhas delineou o estudo, realizou a análise dos dados, participou na redação e aprovou a versão final; RB Sinimbu realizou a revisão bibliográfica, participou na redação e aprovou a versão final; DC Malta, MMA Silva, AF Santos, MLFP Vieira e CL Szwarcwald prestaram contribuições substanciais ao manuscrito, fizeram a revisão e aprovaram a versão final. 


\section{Referências}

1. Abath MB, Leal MCC, Melo Filho DA, Marques APO. Physical abuse of older people reported at the Institute of Forensic Medicine in Recife, Pernambuco State, Brazil. Cad Saude Publica 2010; 26(9):1797-1806.

2. Souto RQ, Araújo FKCD, Cavalcanti AL. Violência sexual: análise de dados relacionados ao atentado violento ao pudor. Rev Bras Promoç Saúde 2012; 25(2):235242.

3. Carvajal FU, Fernádez RR, Abreu RLV. Violencia doméstica en la tercera edad. Rev Cuba Salud Pública 2010; 36(2):189-191.

4. Souto RQ, Leite CCS, França ISX, Cavalcanti AL. Violência sexual contra mulheres portadoras de necessidades especiais: perfil da vítima e do agressor. Cogitare Enferm 2012; 17(1):72-77.

5. Sanches PARA, Lebrão ML, Duarte YAO. Violência contra idosos: uma questão nova? Saúde Soc 2008; 17(3):90-100.

6. Oliveira MLC, Gomes ACG, Amaral CPM, Santos LB. Características dos idosos vítimas de violência doméstica no Distrito Federal. Rev Bras Geriatr Gerontol 2012; 15(3):555-566.

7. Lucena KDT, Silva ATMC, Moraes RM, Silva CC, Bezerra IMP. Análise espacial da violência doméstica contra a mulher entre os anos de 2002 e 2005 em João Pessoa, Paraíba, Brasil. Cad Saude Publica 2012; 28(6):1111-1121.

8. Lindner SR, Coelho EBS, Bolsoni CC, Rojas PF, Boing AF. Prevalência de violência física por parceiro íntimo em homens e mulheres de Florianópolis, Santa Catarina, Brasil: estudo de base populacional. Cad Saude Publica 2015; 31(4):815-826.

9. Silva RA, Araújo TVB, Valongueiro S, Ludermir AB. Enfrentamento da violência infligida pelo parceiro íntimo por mulheres em área urbana da região Nordeste do Brasil. Rev Saude Publica 2012; 46(6):1014-1022.

10. Instituto Brasileiro de Geografia e Estatística (IBGE). Pesquisa Nacional de Saúde, 2013. Rio de Janeiro: IBGE; 2014.

11. Souza-Júnior PRB, Freitas MPS, Antonaci GA, Szwarcwald CL. Desenho da amostra da Pesquisa Nacional de Saúde 2013. Epidemiol Serv Saúde 2015; 24(2):207216.

12. Reichenheim ME, Moraes CL, Szklo A, Hasselmann $\mathrm{MH}$, Souza ER, Lozana JA, Figueiredo V. The magnitude of intimate partner violence in Brazil: portraits from 15 capital cities and the Federal District. Cad Saude Publica 2006; 22(2):425-437.

13. Schraiber LB, D’Oliveira AFPL, França-Júnior I, Diniz S, Portella AP, Ludermir AB, Valença O, Couto MT. Prevalência da violência contra a mulher por parceiro íntimo em regiões do Brasil. Rev Saude Publica 2007; 41(5):797-807.

14. Duarte MC, Fonseca RMGS, Souza V, Pena ED. Gender and violence against women in nursing literature: a review. Rev Bras Enferm 2015; 68(2):325-332.
15. Brasil. Lei $\mathrm{n}^{\circ} 11.340$, de 7 de agosto de 2006. Cria mecanismos para coibir a violência doméstica e familiar contra a mulher, nos termos do $\$ 8^{\circ}$ do art. 226 da Constituição Federal, da Convenção sobre a Eliminação de Todas as Formas de Discriminação contra as Mulheres e da Convenção Interamericana para Prevenir, Punir e Erradicar a Violência contra a Mulher; dispõe sobre a criação dos Juizados de Violência Doméstica e Familiar contra a Mulher; altera o Código de Processo Penal, o Código Penal e a Lei de Execução Penal; e dá outras providências. Diário Oficial da União 2006; 8 ago.

16. Meneghel SN, Mueller B, Collaziol ME, Quadros MM. Repercussões da Lei Maria da Penha no enfrentamento da violência de gênero. Cien Saude Colet 2013; 18(3):691-700.

17. Pasinato W. Oito anos de Lei Maria da Penha: entre avanços, obstáculos e desafios. Rev Estud Fem 2015; 23(2):533-545

18. Rodrigues CS, Malta DC, Godinho T, Mascarenhas MDM, Silva MMA, Silva RE. Acidentes e violências entre mulheres atendidas em seriviços de emergência centinela - Brasil, 2009. Cien Saude Colet 2012; 17(9):2319-2329.

19. Florêncio MVL, Ferreira Filha MO, Sá LD. A violência contra o idoso: dimensão ética e política de uma problemática em ascensão. Rev Eletrônica Enferm 2007; 9(3):847-857.

20. Campos MR, Von Doellinger VR, Mendes LVP, Costa MFS, Pimentel TG, Schramm JMA. Diferenciais de morbimortalidade por causas externas: resultados do estudo Carga Global de Doenças no Brasil, 2008. Cad Saude Publica 2015; 31(1):121-136.

21. Facuri CO, Fernandes AMS, Oliveira KD, Andrade TS, Azevedo RCS. Violência sexual: estudo descritivo sobre as vítimas e o atendimento em um serviço universitário de referência no Estado de São Paulo, Brasil. Cad Saude Publica 2013; 29(5):889-898.

22. Mascarenhas MDM, Andrade SSCA, Neves ACM, Pedrosa AAG, Silva MMA, Malta DC. Violência contra a pessoa idosa: análise das notifiações realizadas no setor saúde - Brasil, 2010. Cien Saude Colet 2012; 17(9):2331-2341.

Artigo apresentado em 20/09/2015

Aprovado em 30/03/2016

Versão final apresentada em 01/04/2016 
